CUBO A Mathematical Journal

Vol.12, № 03, (121-138). October 2010

\title{
Calculations in New Sequence Spaces and Application to Statistical Convergence
}

\author{
Bruno de Malafosse \\ LMAH Université du Havre, BP 4006 IUT Le Havre, \\ 76610 Le Havre. France \\ email: bdemalaf@wanadoo.fr \\ AND \\ VLADIMIR RAKOČEVIĆ ${ }^{1}$ \\ Department of Mathematics, University of Niš, \\ Videgradska 33, 18000 Niš, Serbia \\ email: vrakoc@bankerinter.net
}

\begin{abstract}
In this paper we recall recent results that are direct consequences of the fact that $\left(w_{\infty}(\lambda), w_{\infty}(\lambda)\right)$ is a Banach algebra. Then we define the set $W_{\tau}=D_{\tau} w_{\infty}$ and characterize the sets $W_{\tau}(A)$ where $A$ is either of the operators $\Delta, \Sigma, \Delta(\lambda)$, or $C(\lambda)$. Afterwards we consider the sets $\left[A_{1}, A_{2}\right]_{W_{\tau}}$ of all sequences $X$ such that $A_{1}(\lambda)\left(\left|A_{2}(\mu) X\right|\right) \in W_{\tau}$ where $A_{1}$ and $A_{2}$ are of the form $C(\xi), C^{+}(\xi), \Delta(\xi)$, or $\Delta^{+}(\xi)$ and it is given necessary conditions to get $\left[A_{1}(\lambda), A_{2}(\mu)\right]_{W_{\tau}}$ in the form $W_{\xi}$. Finally we apply the previous results to statistical convergence. So we have conditions to have $x_{k} \rightarrow L(S(A))$ where $A$ is either of the infinite matrices $D_{1 / \tau} C(\lambda) C(\mu), D_{1 / \tau} \Delta(\lambda) \Delta(\mu), D_{1 / \tau} \Delta(\lambda) C(\mu)$. We also give conditions to have $x_{k} \rightarrow 0(S(A))$ where $A$ is either of the operators $D_{1 / \tau} C^{+}(\lambda) \Delta(\mu), D_{1 / \tau} C^{+}(\lambda) C(\mu)$, $D_{1 / \tau} C^{+}(\lambda) C^{+}(\mu)$, or $D_{1 / \tau} \Delta(\lambda) C^{+}(\mu)$.
\end{abstract}

\footnotetext{
${ }^{1}$ Supported by Grant No. 144003 of the Ministry of Science, Technology and Development, Republic of Serbia.
} 


\section{RESUMEN}

Recordamos resultados recientes que son consecuencia directa del hecho de que $\left(w_{\infty}(\lambda)\right.$, $\left.w_{\infty}(\lambda)\right)$ es una algebra de Banach. Entonces nosotros definimos el conjunto $W_{\tau}=D_{\tau} w_{\infty}$ y caracterizamos los conjuntos $W_{\tau}(A)$ donde $A$ es uno de los siguientes operadores $\Delta, \Sigma$, $\Delta(\lambda)$, o $C(\lambda)$. Después consideramos los conjuntos $\left[A_{1}, A_{2}\right]_{W_{\tau}}$ de todas las sucesiones $X$ tal que $A_{1}(\lambda)\left(\left|A_{2}(\mu) X\right|\right) \in W_{\tau}$ donde $A_{1}$ y $A_{2}$ son de la forma $C(\xi), C^{+}(\xi), \Delta(\xi)$, o $\Delta^{+}(\xi)$ y son dadas condiciones necesarias para obtener $\left[A_{1}(\lambda), A_{2}(\mu)\right]_{W_{\tau}}$ en la forma $W_{\xi}$. Finalmente, aplicamos los resultados previos para tener $x_{k} \rightarrow L(S(A))$ donde $A$ es una de las matrices infinitas $D_{1 / \tau} C(\lambda) C(\mu), D_{1 / \tau} \Delta(\lambda) \Delta(\mu), D_{1 / \tau} \Delta(\lambda) C(\mu)$. Nosotros también damos condiciones para tener $x_{k} \rightarrow 0(S(A))$ donde $A$ es uno de los operadores $D_{1 / \tau} C^{+}(\lambda) \Delta(\mu)$, $D_{1 / \tau} C^{+}(\lambda) C(\mu), D_{1 / \tau} C^{+}(\lambda) C^{+}(\mu)$, o $D_{1 / \tau} \Delta(\lambda) C^{+}(\mu)$.

Key words and phrases: Banach algebra, statistical convergence, A-statistical convergence, infinite matrix.

Math. Subj. Class.: 40C05, 40F05, 40J05, 46A15.

\section{Introduction}

In this paper we consider spaces generalizing the well-known sets $w^{0}$ and $w_{\infty}$ introduced and studied by Maddox $[12,13]$. Recall that $w^{0}$ and $w_{\infty}$ are the sets of strongly summable and strongly bounded sequences. In [15] Malkowsky and Rakočević gave characterizations of matrix maps between $w^{0}, w$, or $w_{\infty}$ and $w_{\infty}^{p}$ and between $w^{0}, w$, or $w_{\infty}$ and $l_{1}$. In [2] de Malafosse defined the spaces $w_{\alpha}(\lambda), w_{\alpha}^{(c)}(\lambda)$ and $w_{\alpha}^{0}(\lambda)$ of all sequences that are $\alpha-$ strongly bounded, summable and summable to zero respectively. For instance recall that $w_{\alpha}(\lambda)$ is the set of all sequences $\left(x_{n}\right)_{n}$ such that $1 / \lambda_{n} \sum_{m=1}^{n}\left|x_{m}\right|=\alpha_{n} O(1)$ as $n$ tends to infinity. It was shown that these spaces can be written in the form $s_{\xi}, s_{\xi}^{(c)}$ and $s_{\xi}^{0}$ under some condition on $\alpha$ and $\lambda$.

More recently in [5] it was shown that if $\lambda$ is a sequence exponentially bounded then $\left(w_{\infty}(\lambda), w_{\infty}(\lambda)\right)$ is a Banach algebra. This result led to consider bijective operators mapping between $w_{\infty}(\lambda)$. Here we will use these results to study sets of the form $W_{\tau}=D_{\tau} w_{\infty}, W_{\tau}(\Delta(\lambda)$ ), $W_{\tau}(C(\lambda))$ and $W_{\tau}\left(C^{+}(\lambda)\right)$ generalizing the well-known set of strongly bounded sequences $c_{\infty}=$ $w_{\infty}(\Delta(\mu))$ where $\mu_{n}=n$ for all $n$. These results lead to the study of statistical convergence which was introduced by Steinhaus in 1949, see [16], and studied by several authors such as Fast [7], Fridy, Orhan [8-11] and Connor. Here we will deal with the notion of $A-$ statistical convergence which generalizes the notion of statistical convergence, see [6], where $A$ belongs to a special class of operators. 
The paper is organized as follows. In Section 2 among other things we recall a recent result on the operators $\Delta_{\rho}$ and $\Delta_{\rho}^{T}$ considered as map from $w_{\infty}(\lambda)$ to itself. In Sections 3 and 4 our aim is to give necessary conditions to have $W_{\tau}(A)$ in the form $W_{\xi}$ when $A$ is either one of the matrices $\Delta(\lambda), C(\lambda)$ or $C^{+}(\lambda)$. Then we consider spaces generalizing the wellknown set of all strongly bounded sequences $[C, \Delta]=c_{\infty}$ defined and studied by Maddox. Then we will define the sets $\left[A_{1}, A_{2}\right]_{W_{\tau}}$ of all sequences $X$ with $A_{1}(\lambda)\left(\left|A_{2}(\mu) X\right|\right) \in W_{\tau}$ where $A_{1}$ and $A_{2}$ are of the form $C(\xi), C^{+}(\xi), \Delta(\xi)$, or $\Delta^{+}(\xi)$ and we will give necessary conditions to get $\left[A_{1}(\lambda), A_{2}(\mu)\right]$ in the form $W_{\tau}$. In Section 5 we apply these results to $A$ - statistical convergence, where $A$ is equal to $D_{1 / \tau} A_{1} A_{2}$ and $A_{1}, A_{2}$ are of the form $C(\xi), \Delta(\xi), \Delta(\mu)$, or $C^{+}(\xi)$.

\section{Well Known Results}

For a given infinite matrix $A=\left(a_{n m}\right)_{n, m \geq 1}$ we define the operators $A_{n}$ for any integer $n \geq 1$, by

$$
A_{n}(X)=\sum_{m=1}^{\infty} a_{n m} x_{m}
$$

where $X=\left(x_{n}\right)_{n \geq 1}$, the series intervening in the second member being convergent. So we are led to the study of the infinite linear system

$$
A_{n}(X)=b_{n} \quad n=1,2, \ldots
$$

where $B=\left(b_{n}\right)_{n \geq 1}$ is a one-column matrix and $X$ the unknown, see [2-5]. The equations (2) can be written in the form $A X=B$, where $A X=\left(A_{n}(X)\right)_{n \geq 1}$. In this paper we shall also consider $A$ as an operator from a sequence space into another sequence space.

We will write $s$ for the set of all complex sequences and $\ell_{\infty}$ for the set of all bounded sequences.

Let $E$ and $F$ be any subsets of $s$. When $A$ maps $E$ into $F$ we write that $A \in(E, F)$. So for every $X \in E, A X \in F,\left(A X \in F\right.$ means that for each $n \geq 1$ the series defined by $y_{n}=$ $\sum_{m=1}^{\infty} a_{n m} x_{m}$ is convergent and $\left.\left(y_{n}\right)_{n \geq 1} \in F\right)$.

Body Math For any subset $E$ of $s$, we put

Body Math

$$
A E=\{Y \in s: Y=A X \text { for some } X \in E\}
$$

If $F$ is a subset of $s$, we shall denote

$$
F(A)=F_{A}=\{X \in s: Y=A X \in F\} .
$$


In all what follows we will use the set

$$
U^{+}=\left\{\left(u_{n}\right)_{n \geq 1} \in s: \quad u_{n}>0 \text { for all } n\right\}
$$

and the notation $e=(1, \ldots, 1, \ldots)$. So for $\lambda=\left(\lambda_{n}\right)_{n \geq 1} \in U^{+}$we will consider the sets of strongly bounded and strongly summable sequences, respectively, that is

$$
\begin{aligned}
& w_{\infty}(\lambda)=\left\{X=\left(x_{n}\right)_{n \geq 1} \in s: \sup _{n} \frac{1}{\lambda_{n}} \sum_{m=1}^{n}\left|x_{m}\right|<\infty\right\}, \\
& w^{0}(\lambda)=\left\{X=\left(x_{n}\right)_{n \geq 1} \in s: \lim _{n \rightarrow \infty} \frac{1}{\lambda_{n}} \sum_{m=1}^{n}\left|x_{m}\right|=0\right\}
\end{aligned}
$$

and

$$
w(\lambda)=\left\{X=\left(x_{n}\right)_{n \geq 1} \in s: X-l e \in w^{0}(\lambda) \text { for some } l \in \mathbb{C}\right\}
$$

were studied by Malkowsky, with the concept of exponentially bounded sequences, see [3]. Recall that Maddox [12,13], defined and studied the sets $w_{\infty}(\lambda)=w_{\infty}, w_{0}(\lambda)=w^{0}$ and $w(\lambda)=$ $w$ where $\lambda_{n}=n$ for all $n$.

A Banach space $E$ of complex sequences with the norm \|\|$_{E}$ is a BK space if each projection $P_{n}: X \mapsto P_{n} X=x_{n}$ is continuous. A BK space $E$ is said to have $A K$ if every sequence $X=$ $\left(x_{n}\right)_{n \geq 1} \in E$ has a unique representation $X=\sum_{n=1}^{\infty} x_{n} e_{n}$ where $e_{n}$ is the sequence with 1 in the $\mathrm{n}$-th position and 0 otherwise.

Recall that a nondecreasing sequence $\lambda=\left(\lambda_{n}\right)_{n \geq 1} \in U^{+}$is exponentially bounded if there is an integer $m \geq 2$ such that for all non-negative integers $v$ there is at least one term $\lambda_{n} \in I_{m}^{(v)}=\left[m^{v}, m^{v+1}-1\right]$. It was shown (cf. [14, Lemma 1]) that a non-decreasing sequence $\lambda=\left(\lambda_{n}\right)_{n \geq 1}$ is exponentially bounded if and only if there are reals $s \leq t$ such that for some subsequence $\left(\lambda_{n_{i}}\right)_{i \geq 1}$

$$
0<s \leq \frac{\lambda_{n_{i}}}{\lambda_{n_{i+1}}} \leq t<1 \text { for all } i=1,2, \ldots
$$

such a sequence is called an associated subsequence. Consider now the norm

$$
\|X\|_{\lambda}=\sup _{n}\left(\frac{1}{\lambda_{n}} \sum_{m=1}^{n}\left|x_{m}\right|\right)
$$

In [5] it was shown that if $\lambda=\left(\lambda_{n}\right)_{n \geq 1} \in U^{+}$is exponentially bounded the class $\left(w_{\infty}(\lambda), w_{\infty}(\lambda)\right)$ is a Banach algebra with the norm

$$
\|A\|_{\left(w_{\infty}(\lambda), w_{\infty}(\lambda)\right)}=\sup _{X \neq 0}\left(\frac{\|A X\|_{\lambda}}{\|X\|_{\lambda}}\right) .
$$


For $\rho=\left(\rho_{n}\right)_{n \geq 1}$ consider now the following matrices

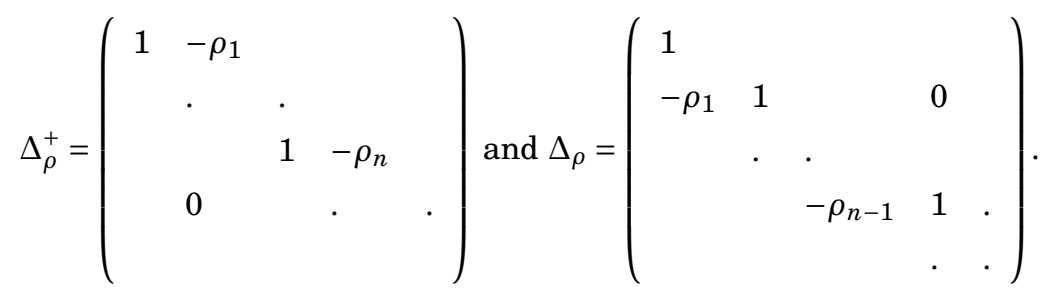

It can easily be shown that if $\rho=\left(\rho_{n}\right)_{n \geq 1}$ and $\left(\lambda_{n+1} / \lambda_{n}\right)_{n \geq 1} \in \ell_{\infty}$ then $\Delta_{\rho}^{+} \in\left(w_{\infty}(\lambda), w_{\infty}(\lambda)\right)$. We also see that $\Delta_{\rho} \in\left(w_{\infty}(\lambda), w_{\infty}(\lambda)\right)$ for $\rho,\left(\lambda_{n-1} / \lambda_{n}\right)_{n \geq 2} \in \ell_{\infty}$. Recall the next result which is a direct consequence of [5, Theorem 5.1 and Theorem 5.12].

Lemma 2.1. Let $\lambda \in U^{+}$be a sequence exponentially bounded.

(i) If

$$
\varlimsup_{n \rightarrow \infty}\left(\frac{\lambda_{n+1}}{\lambda_{n}}\right)<\infty \text { and } \varlimsup_{n \rightarrow \infty}\left|\rho_{n}\right|<\frac{1}{\varlimsup_{n \rightarrow \infty}\left(\frac{\lambda_{n+1}}{\lambda_{n}}\right)},
$$

for given $B \in w_{\infty}(\lambda)$ the equation $\Delta_{\rho}^{+} X=B$ has a unique solution in $w_{\infty}(\lambda)$.

(ii) If

$$
\varlimsup_{n \rightarrow \infty}\left|\rho_{n}\right|<\frac{1}{\varlimsup_{n \rightarrow \infty}\left(\frac{\lambda_{n-1}}{\lambda_{n}}\right)},
$$

then for any given $B \in w_{\infty}(\lambda)$ the equation $\Delta_{\rho} X=B$ has a unique solution in $w_{\infty}(\lambda)$.

When $\lambda$ is a strictly increasing sequence tending to infinity we obtain similar results on the Banach algebra $\left(w^{0}(\lambda), w^{0}(\lambda)\right)$ with the norm $\|A\|_{\left(w_{\infty}(\lambda), w_{\infty}(\lambda)\right)}$.

\section{On the Sets $W_{\tau}(A)$ Where $A$ is Either $\Delta(\lambda), C(\lambda)$ or $C^{+}(\lambda)$}

In the following we will use the operators represented by $C(\lambda)$ and $\Delta(\lambda)$. Let $U$ be the set of all sequences $\left(u_{n}\right)_{n \geq 1}$ with $u_{n} \neq 0$ for all $n$. We define $C(\lambda)$ for $\lambda=\left(\lambda_{n}\right)_{n \geq 1} \in U$, by

$$
[C(\lambda)]_{n m}= \begin{cases}\frac{1}{\lambda_{n}} & \text { if } m \leq n, \\ 0 & \text { otherwise. }\end{cases}
$$

We will write $C(\lambda)^{T}=C^{+}(\lambda), C(e)=\Sigma, \Sigma^{+}=\Sigma^{T}$, and for $\lambda_{n}=n$, the matrix $C_{1}=C\left((n)_{n}\right)$ is called the Cesaro operator. If It can be proved that the matrix $\Delta(\lambda)$ with

$$
[\Delta(\lambda)]_{n m}= \begin{cases}\lambda_{n} & \text { if } m=n, \\ -\lambda_{n-1} & \text { if } m=n-1 \text { and } n \geq 2 \\ 0 & \text { otherwise }\end{cases}
$$


is the inverse of $C(\lambda)$, see [2,3]. We will use the following sets

$$
\begin{aligned}
\Gamma & =\left\{X \in U^{+}: \varlimsup_{n \rightarrow \infty}\left(\frac{x_{n-1}}{x_{n}}\right)<1\right\}, \\
\Gamma^{+} & =\left\{X \in U^{+}: \varlimsup_{n \rightarrow \infty}\left(\frac{x_{n+1}}{x_{n}}\right)<1\right\} .
\end{aligned}
$$

Note that $X \in \Gamma^{+}$if and only if $1 / X \in \Gamma$.

For given sequence $\tau=\left(\tau_{n}\right)_{n \geq 1} \in U^{+}$, we write $D_{\tau}$ for the diagonal matrix defined by $\left[D_{\tau}\right]_{n n}=\tau_{n}$ for all $n$. For any subset $E$ of $s$, we write

$$
D_{\tau} E=\left\{X=\left(x_{n}\right)_{n \geq 1} \in s:\left(\frac{x_{n}}{\tau_{n}}\right)_{n} \in E\right\} .
$$

We put $W_{\tau}=D_{\tau} w_{\infty}$ for $\tau \in U^{+}$, that is

$$
W_{\tau}=\left\{X:\|X\|_{W_{\tau}}=\sup _{n}\left(\frac{1}{n} \sum_{m=1}^{\infty} \frac{\left|x_{m}\right|}{\tau_{m}}\right)<\infty\right\} .
$$

It can easily be seen that $W_{\tau}=w_{\infty}\left(D_{1 / \tau}\right)$ is a BK space with norm \|\|$_{W_{\tau}}$, (cf. [17, Theorem 4.3.6, p. 52]). In all that follows we will use the convention that the entries with subscripts strictly less than 1 are equal to zero. Then we are interested in the study of the following sets where $\lambda, \tau \in U^{+}$.

$$
\begin{aligned}
W_{\tau}(\Delta(\lambda)) & =\left\{X: \sup _{n}\left(\frac{1}{n} \sum_{m=1}^{n} \frac{1}{\tau_{m}}\left|\lambda_{m} x_{m}-\lambda_{m-1} x_{m-1}\right|\right)<\infty\right\}, \\
W_{\tau}(C(\lambda)) & =\left\{X: \sup _{n} \frac{1}{n} \sum_{m=1}^{n}\left(\frac{1}{\lambda_{m} \tau_{m}} \sum_{k=1}^{m}\left|x_{k}\right|\right)<\infty\right\}, \\
W_{\tau}\left(C^{+}(\lambda)\right) & =\left\{X: \sup _{n} \frac{1}{n} \sum_{m=1}^{n}\left(\frac{1}{\tau_{m}} \sum_{k=m}^{\infty} \frac{\left|x_{k}\right|}{\lambda_{k}}\right)<\infty\right\} .
\end{aligned}
$$

Note that for $\lambda_{n}=n$ and $\tau=e, W_{\tau}(\Delta(\lambda))$ is the well known set of all strongly and bounded sequences $c_{\infty}$. We obtain the following result that is a direct consequence of Lemma 2.1.

Proposition 3.1. (i) If $\tau \in \Gamma$ then the operators $\Delta$ and $\Sigma$ are bijective from $W_{\tau}$ into itself and

$$
W_{\tau}(\Delta)=W_{\tau}, W_{\tau}(\Sigma)=W_{\tau} .
$$

(ii) a) If $\lambda \tau \in \Gamma$ then

$$
W_{\tau}(C(\lambda))=W_{\lambda \tau}
$$

b) If $\tau \in \Gamma$ then

$$
W_{\tau}(\Delta(\lambda))=W_{\tau / \lambda}
$$


(iii) Let $\tau \in \Gamma^{+}$. Then

a) the operators $\Delta^{+}$and $\Sigma^{+}$are bijective from $W_{\tau}$ into itself and

$$
W_{\tau}\left(\Sigma^{+}\right)=W_{\tau} .
$$

b) the operator $C^{+}(\lambda)$ is bijective from $W_{\lambda \tau}$ into $W_{\tau}$ and

$$
W_{\tau}\left(C^{+}(\lambda)\right)=W_{\lambda \tau} .
$$

Proof. (i) By Lemma 2.1 where $\rho_{n}=\tau_{n-1} / \tau_{n}$ and $\lambda_{n}=n$ for all $n$, we easily see that if

$$
\varlimsup_{n \rightarrow \infty} \frac{\tau_{n-1}}{\tau_{n}}<\frac{1}{\lim _{n \rightarrow \infty}\left(\frac{n-1}{n}\right)}=1,
$$

that is $\tau \in \Gamma$, then $D_{1 / \tau} \Delta D_{\tau}$ is bijective from $w_{\infty}$ to itself. This means that $\Delta$ is bijective from $D_{\tau} w_{\infty}$ to itself. Since $\Sigma$ is also bijective from $D_{\tau} w_{\infty}$ to itself, this shows $W_{\tau}(\Delta)=W_{\tau}$ and $W_{\tau}(\Sigma)=W_{\tau}$.

(ii) We have $X \in W_{\tau}(C(\lambda))$ if and only if $\Sigma X \in D_{\lambda \tau} w_{\infty}=W_{\lambda_{\tau}}$. This means that $X \in W_{\lambda_{\tau}}(\Sigma)$ and by (i) the condition $\lambda \tau \in \Gamma$ implies $W_{\lambda \tau}(\Sigma)=W_{\lambda \tau}$. Then $W_{\tau}(C(\lambda))=W_{\lambda \tau}$ and $C(\lambda)$ is bijective from $W_{\lambda \tau}$ to $W_{\tau}$. Since $\Delta(\lambda)=C(\lambda)^{-1}$ we conclude $\Delta(\lambda)$ bijective from $W_{\tau}$ to $W_{\lambda \tau}$ and $W_{\lambda \tau}(\Delta(\lambda))=W_{\tau}$. We deduce that for $\tau \in \Gamma, W_{\tau}(\Delta(\lambda))=W_{\tau / \lambda}$.

(iii) a) By Lemma 2.1 with $\rho_{n}=\tau_{n+1} / \tau_{n}$ and $\lambda_{n}=n$ we have $\Delta_{\rho}^{+}=D_{1 / \tau} \Delta^{+} D_{\tau}$ and $\Delta^{+}$is bijective from $D_{\tau} w_{\infty}=W_{\tau}$ into itself for $\tau \in \Gamma^{+}$and it is the same for $\Sigma^{+}$. Now the equation $\Sigma^{+} X=Y$ for $Y \in W_{\tau}$ is equivalent to

$$
\sum_{m=n}^{\infty} x_{m}=y_{n} \text { for all } n .
$$

We deduce (8) has a unique solution $X=\left(y_{n}-y_{n+1}\right)_{n \geq 1}=\Delta^{+} Y \in W_{\tau}$ and $W_{\tau}\left(\Sigma^{+}\right)=W_{\tau}$.

b) We have

$$
W_{\tau}\left(C^{+}(\lambda)\right)=\left\{X: \Sigma^{+} D_{1 / \lambda} X \in W_{\tau}\right\}=D_{\lambda} W_{\tau}\left(\Sigma^{+}\right) .
$$

Now as we have seen above since $\tau \in \Gamma^{+}$we get $W_{\tau}\left(\Sigma^{+}\right)=W_{\tau}$ and

$$
W_{\tau}\left(C^{+}(\lambda)\right)=D_{\lambda} W_{\tau}\left(\Sigma^{+}\right)=D_{\lambda} W_{\tau}=W_{\lambda \tau} .
$$

This gives the conclusion. 


\section{Calculations in New Sequence Spaces}

4.1 The sets $[C, \Delta]_{W_{\tau}},[C, C]_{W_{\tau}},\left[C^{+}, \Delta\right]_{W_{\tau}},\left[C^{+}, C\right]_{W_{\tau}}$ and $\left[C^{+}, C^{+}\right]_{W_{\tau}}$.

In [4], were defined and studied the sets

$$
\left[A_{1}, A_{2}\right]=\left[A_{1}(\lambda), A_{2}(\mu)\right]=\left\{X \in s: A_{1}(\lambda)\left(\left|A_{2}(\mu) X\right|\right) \in D_{\tau} l_{\infty}\right\}
$$

where $|X|=\left(\left|x_{n}\right|\right)_{n \geq 1}, A_{1}$ and $A_{2}$ of the form $C(\xi), C^{+}(\xi), \Delta(\xi)$, or $\Delta^{+}(\xi)$ for $\xi \in U^{+}$. It was given necessary conditions to get $\left[A_{1}(\lambda), A_{2}(\mu)\right]$ in the form $s_{\gamma}$.

Similarly in the following we will put

$$
\left[A_{1}, A_{2}\right]_{W_{\tau}}=\left[A_{1}(\lambda), A_{2}(\mu)\right]_{W_{\tau}}=\left\{X \in s: A_{1}(\lambda)\left(\left|A_{2}(\mu) X\right|\right) \in W_{\tau}\right\}
$$

for $\lambda, \mu, \tau \in U^{+}$. We can explicitly write the previous sets $\left[A_{1}, A_{2}\right]_{W_{\tau}}$ as follows.

$$
\begin{aligned}
{[C, \Delta]_{W_{\tau}} } & =\left\{X: \sup _{n}\left(\frac{1}{n} \sum_{m=1}^{n} \frac{1}{\lambda_{m} \tau_{m}} \sum_{k=1}^{m}\left|\mu_{k} x_{k}-\mu_{k-1} x_{k-1}\right|\right)<\infty\right\}, \\
{[C, C]_{W_{\tau}} } & =\left\{X: \sup _{n}\left(\frac{1}{n} \sum_{m=1}^{n}\left(\frac{1}{\lambda_{m} \tau_{m}} \sum_{k=1}^{m} \frac{1}{\mu_{k}}\left|\sum_{i=1}^{k} x_{i}\right|\right)\right)<\infty\right\}, \\
{\left[C^{+}, \Delta\right]_{W_{\tau}} } & =\left\{X: \sup _{n}\left(\frac{1}{n} \sum_{m=1}^{n}\left(\frac{1}{\tau_{m}} \sum_{k=m}^{\infty} \frac{1}{\lambda_{k}}\left|\mu_{k} x_{k}-\mu_{k-1} x_{k-1}\right|\right)\right)<\infty\right\}, \\
{\left[C^{+}, C\right]_{W_{\tau}} } & =\left\{X: \sup _{n}\left(\frac{1}{n} \sum_{m=1}^{n}\left(\frac{1}{\tau_{m}} \sum_{k=m}^{\infty} \frac{1}{\lambda_{k}} \frac{1}{\mu_{k}}\left|\sum_{i=1}^{k} x_{i}\right|\right)\right)<\infty\right\}, \\
{\left[C^{+}, C^{+}\right]_{W_{\tau}} } & =\left\{X: \sup _{n}\left(\frac{1}{n} \sum_{m=1}^{n}\left(\frac{1}{\tau_{m}} \sum_{k=m}^{\infty} \frac{1}{\lambda_{k}}\left|\sum_{i=k}^{\infty} \frac{x_{i}}{\mu_{i}}\right|\right)\right)<\infty\right\} .
\end{aligned}
$$

Note that if $\lambda_{n}=\mu_{n}$ for all $n$ we get the well known set of sequences that are strongly bounded $[C, \Delta]_{W_{e}}=c_{\infty}(\lambda)$. We can state the following.

Theorem 4.1. Let $\lambda, \mu, \tau \in U^{+}$.

(i) If $\lambda \tau \in \Gamma$ then

$$
[C, \Delta]_{W_{\tau}}=W_{\lambda \tau / \mu}
$$

(ii) if $\lambda \tau, \lambda \mu \tau \in \Gamma$ then

$$
[C, C]_{W_{\tau}}=W_{\lambda \mu \tau}
$$

(iii) if $\tau \in \Gamma^{+}$and $\lambda \tau \in \Gamma$ then

$$
\left[C^{+}, \Delta\right]_{W_{\tau}}=W_{\lambda \tau / \mu}
$$


(iv) if $\tau \in \Gamma^{+}$and $\lambda \mu \tau \in \Gamma$ then

$$
\left[C^{+}, C\right]_{W_{\tau}}=W_{\lambda \mu \tau}
$$

(v) if $\tau, \lambda \tau \in \Gamma^{+}$then

$$
\left[C^{+}, C^{+}\right]_{W_{\tau}}=W_{\lambda \mu \tau}
$$

Proof. In the following we will use the fact that for any $\xi \in U^{+}$we have $|X| \in W_{\xi}$ if and only if $X \in W_{\xi}$.

(i) We have $C(\lambda)(|\Delta(\mu) X|) \in W_{\tau}$ if and only if $|\Delta(\mu) X| \in W_{\tau}(C(\lambda))$ and by Proposition 3.1, since $\lambda \tau \in \Gamma$ we get $W_{\tau}(C(\lambda))=W_{\lambda \tau}$. Then by Proposition 3.1 (ii) we have $W_{\lambda \tau}(\Delta(\mu))=W_{\lambda \tau / \mu}$ and we conclude $\Delta(\mu) X \in W_{\lambda \tau}$ if and only if $X \in W_{\lambda \tau}(\Delta(\mu))=W_{\lambda \tau / \mu}$, that is $[C, \Delta]_{W_{\tau}}=W_{\lambda \tau / \mu}$.

(ii) Here we have $C(\lambda)(|C(\mu) X|) \in W_{\tau}$ if and only if $|C(\mu) X| \in W_{\tau}(C(\lambda))$; and since $\lambda \tau \in \Gamma$ by Proposition 3.1 we have $W_{\tau}(C(\lambda))=W_{\lambda \tau}$. So $X \in[C, C]_{W_{\tau}}$ if and only if $C(\mu) X \in W_{\lambda \tau}$, that is $X \in W_{\lambda \tau}(C(\mu))$. Then by Proposition 3.1 (ii) a) $\lambda \mu \tau \in \Gamma$ implies $W_{\lambda \tau}(C(\mu))=W_{\lambda \mu \tau}$ and we have shown (ii).

(iii) For any given $X \in\left[C^{+}, \Delta\right]_{W_{\tau}}$ we have $\Delta(\mu) X \in W_{\tau}\left(C^{+}(\lambda)\right)$ and for $\tau \in \Gamma^{+}$we have $W_{\tau}\left(C^{+}(\lambda)\right)=W_{\lambda \tau}$. Now the condition $\lambda \tau \in \Gamma$ implies $X \in\left[C^{+}, \Delta\right]_{W_{\tau}}$ if and only if $X \in W_{\lambda \tau}(\Delta(\mu))=$ $W_{\lambda \tau / \mu}$ and we have shown (iii).

(iv) Let $X \in\left[C^{+}, C\right]_{W_{\tau}}$. We have $\tau \in \Gamma^{+}$implies $W_{\tau}\left(C^{+}(\lambda)\right)=W_{\lambda \tau}$ and so $X \in\left[C^{+}, C\right]_{W_{\tau}}$ if and only if $C(\mu) X \in W_{\lambda \tau}$. Now since $\lambda \mu \tau \in \Gamma$ we have $W_{\lambda \tau}(C(\mu))=W_{\lambda \mu \tau}$ and we conclude $\left[C^{+}, C\right]_{W_{\tau}}=W_{\lambda \mu \tau}$.

(v) As above $X \in\left[C^{+}, C^{+}\right]_{W_{\tau}}$ if and only if $C^{+}(\mu) X \in W_{\tau}\left(C^{+}(\lambda)\right)$ and the condition $\tau \in \Gamma^{+}$ implies $W_{\tau}\left(C^{+}(\lambda)\right)=W_{\lambda \tau}$. Since $\lambda \tau \in \Gamma^{+}$we conclude $W_{\lambda \tau}\left(C^{+}(\mu)\right)=W_{\lambda \mu \tau}$ that is $\left[C^{+}, C^{+}\right]_{W_{\tau}}=$ $W_{\lambda \mu \tau}$.

Now we are led to study sets of the form $\left[\Delta, A_{2}\right]_{W_{\tau}}$ for $A_{2} \in\left\{\Delta, \Delta, C^{+}\right\}$.

\subsection{The sets $[\Delta, \Delta]_{W_{\tau}},[\Delta, C]_{W_{\tau}}$ and $\left[\Delta, C^{+}\right]_{W_{\tau}}$}

Using the convention $\mu_{0}=0$, and the notation $\Delta(\mu) x_{m}=\mu_{m} x_{m}-\mu_{m-1} x_{m-1}$ for $m \geq 1$ we explicitly have

$$
\begin{aligned}
{[\Delta, \Delta]_{W_{\tau}} } & =\left\{X: \sup _{n}\left(\frac{1}{n} \sum_{m=1}^{n} \frac{1}{\tau_{m}}\left|\lambda_{m}\right| \Delta(\mu) x_{m}\left|-\lambda_{m-1}\right| \Delta(\mu) x_{m-1}||\right)<\infty\right\}, \\
{[\Delta, C]_{W_{\tau}} } & =\left\{X: \sup _{n}\left(\frac{1}{n} \sum_{m=1}^{n} \frac{1}{\tau_{m}}\left|\lambda_{m}\right| \frac{1}{\mu_{m}} \sum_{k=1}^{m} x_{k}\left|-\lambda_{m-1}\right| \frac{1}{\mu_{m-1}} \sum_{k=1}^{m-1} x_{k}||\right)<\infty\right\},
\end{aligned}
$$




$$
\left[\Delta, C^{+}\right]_{W_{\tau}}=\left\{X: \sup _{n}\left(\frac{1}{n} \sum_{m=1}^{n} \frac{1}{\tau_{m}}\left|\lambda_{m}\right| \sum_{k=m}^{\infty} \frac{x_{k}}{\mu_{k}}\left|-\lambda_{m-1}\right| \sum_{k=m-1}^{\infty} \frac{x_{k}}{\mu_{k}}||\right)<\infty\right\} .
$$

As a direct consequence of Proposition 3.1 we also obtain the following results.

Theorem 4.2. Let $\lambda, \mu, \tau \in U^{+}$. Then

(i) If $\tau, \tau / \lambda \in \Gamma$ then

$$
[\Delta, \Delta]_{W_{\tau}}=W_{\tau / \lambda \mu}
$$

(ii) If $\tau, \tau \mu / \lambda \in \Gamma$ then

$$
[\Delta, C]_{W_{\tau}}=W_{\tau \mu / \lambda} .
$$

(iii) If $\tau, \tau / \lambda \in \Gamma^{+}$then

$$
\left[\Delta, C^{+}\right]_{W_{\tau}}=W_{\tau \mu / \lambda} .
$$

Proof. (i) Let $X \in[\Delta, \Delta]_{W_{\tau}}$. Since $\tau \in \Gamma$ we have $W_{\tau}(\Delta(\lambda))=W_{\tau / \lambda}$ and $\Delta(\lambda)|\Delta(\mu) X| \in W_{\tau}$ means $\Delta(\mu) X \in W_{\tau / \lambda}$. We conclude $W_{\tau / \lambda}(\Delta(\mu))=W_{\tau / \lambda \mu}$ for $\tau / \lambda \in \Gamma$.

(ii) Reasoning as above since $\tau \in \Gamma$ we have $X \in[\Delta, C]_{W_{\tau}}$ if and only if $C(\mu) X \in W_{\tau / \lambda}$. We conclude since the condition $\tau \mu / \lambda \in \Gamma$ implies $W_{\tau / \lambda}(C(\mu))=W_{\tau \mu / \lambda}$.

(iii) Here under the conditions $\tau, \tau / \lambda \in \Gamma^{+}$, we have $X \in\left[\Delta, C^{+}\right]_{W_{\tau}}$ if and only if $X \in$ $W_{\tau / \lambda}\left(C^{+}(\mu)\right)=W_{\tau \mu / \lambda}$.

The previous results can be applied to the case when $w_{\infty}$ is replaced by $w^{0}$.

\subsection{The sets $\left[A_{1}, A_{2}\right]_{W_{\tau}^{0}}$}

Using the Banach algebra $\left(w^{0}(\lambda), w^{0}(\lambda)\right)$ we get similar results to those given above replacing $w_{\infty}(\lambda)$ by $w^{0}(\lambda)$ and $W_{\tau}$ by $W_{\tau}^{0}=D_{\tau} w^{0}$. Note that $X \in W_{\tau}^{0}$ if and only if

$$
\frac{1}{n} \sum_{m=1}^{n} \frac{\left|x_{m}\right|}{\tau_{m}} \rightarrow 0(n \rightarrow \infty)
$$

By $\left[17\right.$, Theorem 4.3.6, p. 52] the set $W_{\tau}^{0}$ is a BK space with AK normed by \|\|$_{W_{\tau}}$. So we can state the following.

Proposition 4.3. Let $\lambda, \mu \in U^{+}$.

(i) If $\lambda \tau \in \Gamma$ then $[C, \Delta]_{W_{\tau}^{0}}=W_{\lambda \tau / \mu}^{0}$;

(ii) if $\lambda \tau, \lambda \mu \tau \in \Gamma$ then $[C, C]_{W_{\tau}^{0}}=W_{\lambda \mu \tau}^{0}$;

(iii) if $\tau \in \Gamma^{+}$and $\lambda \tau \in \Gamma$ then $\left[C^{+}, \Delta\right]_{W_{\tau}^{0}}=W_{\lambda \tau / \mu}^{0}$; 
(iv) if $\tau \in \Gamma^{+}$and $\lambda \mu \tau \in \Gamma$ then $\left[C^{+}, C\right]_{W_{\tau}^{0}}=W_{\lambda \mu \tau}^{0}$;

(v) if $\tau, \lambda \tau \in \Gamma^{+}$then $\left[C^{+}, C^{+}\right]_{W_{\tau}^{0}}=W_{\lambda \mu \tau}^{0}$;

(vi) if $\tau, \tau / \lambda \in \Gamma$ then $[\Delta, \Delta]_{W_{\tau}^{0}}=W_{\tau / \lambda \mu}^{0}$;

(vii) if $\tau, \tau \mu / \lambda \in \Gamma$ then $[\Delta, C]_{W_{\tau}^{0}}=W_{\tau \mu / \lambda}^{0}$;

(viii) if $\tau, \tau / \lambda \in \Gamma^{+}$then $\left[\Delta, C^{+}\right]_{W_{\tau}^{0}}=W_{\tau \mu / \lambda}^{0}$.

We immediatly get the next remark.

Remark 4.4. It can easily be seen that in Proposition 4.3 each of the sets $\left[A_{1}, A_{2}\right]_{W_{T}^{0}}$ is equal to $W_{\tau}^{0}\left(A_{1} A_{2}\right)$. This result is a direct consequence of the previous proofs and of the fact that $W_{\tau}^{0}$ is of absolute type, that is $|X| \in W_{\tau}^{0}$ if and only if $X \in W_{\tau}^{0}$.

These results can be applied to statistical convergence.

\section{Application to $A$-Statistical Convergence}

In this section we will give conditions to have $x_{k} \rightarrow L(S(A))$ where $A$ is either of the infinite matrices $D_{1 / \tau} C(\lambda) C(\mu), D_{1 / \tau} \Delta(\lambda) \Delta(\mu)$, or $D_{1 / \tau} \Delta(\lambda) C(\mu)$. Then we give conditions to have $x_{k} \rightarrow 0(S(A))$ where $A$ is either of the operators $D_{1 / \tau} C^{+}(\lambda) \Delta(\mu), D_{1 / \tau} C^{+}(\lambda) C(\mu), D_{1 / \tau} C^{+}(\lambda)$ $C^{+}(\mu)$ and $D_{1 / \tau} \Delta(\lambda) C^{+}(\mu)$.

The sequence $X=\left(x_{n}\right)_{n \geq 1}$ is said to be statiscally convergent to the number $L$ if

$$
\lim _{n \rightarrow \infty} \frac{1}{n}\left|\left\{k \leq n:\left|x_{k}-L\right| \geq \varepsilon\right\}\right|=0 \text { for all } \varepsilon>0,
$$

where the vertical bars indicate the number of elements in the enclosed set. In this case we will write $x_{k} \rightarrow L(S)$ or $s t-\lim X=L$.

Let $A \in(E, F)$ for given $L \in \mathbb{C}$ and for every $\varepsilon>0$ we will use the notation

$$
I_{\varepsilon}(A)=\left\{k \leq n:\left|[A X]_{k}-L\right| \geq \varepsilon\right\},
$$

(where we assume that every series $[A X]_{k}=A_{k}(X)=\sum_{m=1}^{\infty} a_{k m} x_{m}$ for $k \geq 1$ is convergent). We will say that $X=\left(x_{n}\right)_{n \geq 1}$ is $A$-statistically convergent to $L$ if for every $\varepsilon>0$,

$$
\lim _{n \rightarrow \infty} \frac{1}{n}\left|I_{\varepsilon}(A)\right|=0 \text {. }
$$

Then we will write $x_{k} \rightarrow L(S(A))$ and for $A=I, x_{k} \rightarrow L(S(I))$ means that $s t-\lim X=L$, (cf. [6]).

Now we require a lemma where we will put $T^{-1} e=\widetilde{l}=\left(l_{n}\right)_{n \geq 1}$ for given triangle $T$, that is $T=\left(t_{n m}\right)_{n, m \geq 1}$ with $t_{n n} \neq 0$ and $t_{n m}=0$ if $m>n$ for all $n, m$. 
We can state the following.

Lemma 5.1. If $X-L \widetilde{l} \in w^{0}(T)$ then $x_{k}$ is $T$ - statistically convergent to $L$.

Proof. The condition $X-L \widetilde{l} \in w^{0}(T)$ means that $T(X-L \widetilde{l}) \in w^{0}$. Since

$$
T X-L e=T\left(X-L T^{-1} e\right)=T(X-L \widetilde{l})
$$

for any $\varepsilon>0$ we have

$$
\begin{aligned}
y_{n} & =\frac{1}{n} \sum_{k=1}^{n}\left|[T X]_{k}-L\right|=\frac{1}{n} \sum_{k=1}^{n}\left|[T(X-L \widetilde{l})]_{k}\right| \\
& \geq \frac{1}{n} \sum_{k \in I_{\varepsilon}(T)}\left|[T(X-L \widetilde{l})]_{k}\right| \\
& \geq \frac{1}{n} \sum_{k \in I_{\varepsilon}(T)} \varepsilon \\
& \geq \frac{\varepsilon}{n}\left|\left\{k \leq n:\left|[T X]_{k}-L\right| \geq \varepsilon\right\}\right| .
\end{aligned}
$$

We conclude that $X-L \widetilde{l} \in w^{0}(T)$ implies $y_{n} \rightarrow 0(n \rightarrow \infty)$ and $x_{k} \rightarrow L(S(T))$.

We are led to state the next results.

Theorem 5.2. (i) Let $\lambda \tau, \lambda \tau \mu \in \Gamma$. If

$$
\lim _{n \rightarrow \infty} \frac{1}{n} \sum_{k=1}^{n} \frac{\left|x_{k}-L\left[\lambda_{k} \mu_{k} \tau_{k}+\left(\mu_{k-1}+\mu_{k}\right) \lambda_{k-1} \tau_{k-1}-\lambda_{k-2} \mu_{k-2} \tau_{k-2}\right]\right|}{\lambda_{k} \mu_{k} \tau_{k}}=0
$$

then $x_{k} \rightarrow L\left(S\left(D_{1 / \tau} C(\lambda) C(\mu)\right)\right)$, that is for every $\varepsilon>0$

$$
\lim _{n \rightarrow \infty} \frac{1}{n}\left|\left\{k \leq n:\left|\frac{1}{\lambda_{k} \tau_{k}} \sum_{i=1}^{k} \frac{1}{\mu_{i}}\left(\sum_{j=1}^{i} x_{j}\right)-L\right| \geq \varepsilon\right\}\right|=0 .
$$

(ii) Let $\tau, \tau / \lambda \in \Gamma$. If

$$
\lim _{n \rightarrow \infty} \frac{1}{n} \sum_{k=1}^{n} \frac{\lambda_{k} \mu_{k}}{\tau_{k}}\left|x_{k}-L\left(\frac{1}{\mu_{k}} \sum_{i=1}^{k} \frac{1}{\lambda_{i}} \sum_{j=1}^{i} \tau_{j}\right)\right|=0
$$

then $x_{k} \rightarrow L\left(S\left(D_{1 / \tau} \Delta(\lambda) \Delta(\mu)\right)\right)$, that is for every $\varepsilon>0$

$$
\lim _{n \rightarrow \infty} \frac{1}{n}\left|\left\{k \leq n:\left|\frac{1}{\tau_{k}}\left[\lambda_{k} \Delta(\mu) x_{k}-\lambda_{k-1} \Delta(\mu) x_{k-1}\right]-L\right| \geq \varepsilon\right\}\right|=0 .
$$

(iii) Let $\tau, \tau \mu / \lambda \in \Gamma$. If

$$
\lim _{n \rightarrow \infty} \frac{1}{n} \sum_{k=1}^{n} \frac{\lambda_{k}}{\mu_{k} \tau_{k}}\left|x_{k}-L\left[\left(\frac{\mu_{k}}{\lambda_{k}}-\frac{\mu_{k-1}}{\lambda_{k-1}}\right) \sum_{i=1}^{k-1} \tau_{i}+\frac{\mu_{k}}{\lambda_{k}} \tau_{k}\right]\right|=0
$$


then $x_{k} \rightarrow L\left(S\left(D_{1 / \tau} \Delta(\lambda) C(\mu)\right)\right)$, that is for every $\varepsilon>0$

$$
\lim _{n \rightarrow \infty} \frac{1}{n}\left|\left\{k \leq n:\left|\frac{1}{\tau_{k}}\left[\left(\frac{\lambda_{k}}{\mu_{k}}-\frac{\lambda_{k-1}}{\mu_{k-1}}\right) \sum_{i=1}^{k-1} x_{i}+\frac{\lambda_{k}}{\mu_{k}} x_{k}\right]-L\right| \geq \varepsilon\right\}\right|=0 .
$$

Proof. (i) First by Proposition 4.3 (ii) and Remark 4.4, we easily see that for $\lambda \tau, \lambda \tau \mu \in \Gamma$ we have $W_{\tau}^{0}(C(\lambda) C(\mu))=W_{\lambda \mu \tau}^{0}$. Then putting $T=D_{1 / \tau} C(\lambda) C(\mu)$ we get

$$
w^{0}(T)=W_{\tau}^{0}(C(\lambda) C(\mu))=W_{\lambda \mu \tau}^{0} .
$$

Then $\widetilde{l}=T^{-1} e=\Delta(\mu) \Delta(\lambda) D_{\tau} e$ for each $n$ with

$$
l_{n}=\left[\Delta(\mu) \Delta(\lambda) D_{\tau} e\right]_{n}=\lambda_{n} \mu_{n} \tau_{n}+\left(\mu_{n-1}+\mu_{n}\right) \lambda_{n-1} \tau_{n-1}-\lambda_{n-2} \mu_{n-2} \tau_{n-2}
$$

Using (10) and (11) we see that condition (9) is equivalent $X-L \widetilde{l} \in w^{0}(T)$. We conclude by Lemma 5.1 that $x_{k} \rightarrow L(S(T))$. This completes the proof of (i).

(ii) By Proposition 4.3 (vi) and Remark 4.4, since $\tau, \tau / \lambda \in \Gamma$ we have $W_{\tau}^{0}(\Delta(\lambda) \Delta(\mu))=W_{\tau / \lambda \mu}^{0}$. Then putting $T^{\prime}=D_{1 / \tau} \Delta(\lambda) \Delta(\mu)$ we get

$$
w^{0}\left(T^{\prime}\right)=W_{\tau}^{0}(\Delta(\lambda) \Delta(\mu))=W_{\tau / \lambda \mu}^{0} .
$$

Since $\widetilde{l^{\prime}}=T^{\prime-1} e=C(\mu) C(\lambda) D_{\tau} e$ we have

$$
l_{n}^{\prime}=\left[C(\mu) C(\lambda) D_{\tau} e\right]_{n}=\frac{1}{\mu_{n}} \sum_{i=1}^{n} \frac{1}{\lambda_{i}}\left(\sum_{j=1}^{i} \tau_{j}\right) \text { for all } n .
$$

By Lemma 5.1 we conclude $x_{k} \rightarrow L\left(S\left(D_{1 / \tau} \Delta(\lambda) \Delta(\mu)\right)\right)$ for all $X$ with

$$
\lim _{n \rightarrow \infty} \frac{1}{n} \sum_{k=1}^{n}\left|x_{k}-L l_{k}^{\prime}\right| \frac{\lambda_{k} \mu_{k}}{\tau_{k}}=0
$$

This shows (ii).

(iii) Again by Proposition 4.3 (vii) and Remark 4.4, since $\tau, \tau \mu / \lambda \in \Gamma$ we have $W_{\tau}^{0}(\Delta(\lambda) C(\mu))=$ $W_{\tau \mu / \lambda}^{0}$. Then putting $T^{\prime \prime}=D_{1 / \tau} \Delta(\lambda) C(\mu)$ we get

$$
w^{0}\left(T^{\prime \prime}\right)=W_{\tau}^{0}(\Delta(\lambda) C(\mu))=W_{\tau \mu / \lambda}^{0} .
$$

Writing $\widetilde{l^{\prime \prime}}=T^{\prime \prime}-1 e=\Delta(\mu) C(\lambda) D_{\tau} e$ we successively get

$$
D_{\tau} e=\left(\tau_{n}\right)_{n \geq 1}, C(\lambda) D_{\tau} e=\left(\left(\sum_{i=1}^{n} \tau_{i}\right) / \lambda_{n}\right)_{n \geq 1}
$$

and

$$
\Delta(\mu) C(\lambda) D_{\tau} e=\left(\frac{\mu_{n}}{\lambda_{n}} \sum_{i=1}^{n} \tau_{i}-\frac{\mu_{n-1}}{\lambda_{n-1}} \sum_{i=1}^{n-1} \tau_{i}\right)_{n \geq 1} .
$$


So for each $n$ we have

$$
l_{n}^{\prime \prime}=\left[\Delta(\mu) C(\lambda) D_{\tau} e\right]_{n}=\left(\frac{\mu_{n}}{\lambda_{n}}-\frac{\mu_{n-1}}{\lambda_{n-1}}\right) \sum_{i=1}^{n-1} \tau_{i}+\frac{\mu_{n}}{\lambda_{n}} x_{k} .
$$

We conclude that for every $X$ with

$$
\lim _{n \rightarrow \infty} \frac{1}{n} \sum_{k=1}^{n}\left|x_{k}-L l_{k}^{\prime \prime}\right| \frac{\lambda_{k}}{\mu_{k} \tau_{k}}=0
$$

then $x_{k} \rightarrow L\left(S\left(T^{\prime \prime}\right)\right)$. Finally we easily get

$$
\begin{aligned}
{\left[T^{\prime \prime} X\right]_{n} } & =\frac{1}{\tau_{n}}\left(\frac{\lambda_{n}}{\mu_{n}} \sum_{i=1}^{n} x_{i}-\frac{\lambda_{n-1}}{\mu_{n-1}} \sum_{i=1}^{n-1} x_{i}\right) \\
& =\frac{1}{\tau_{n}}\left[\left(\frac{\lambda_{n}}{\mu_{n}}-\frac{\lambda_{n-1}}{\mu_{n-1}}\right) \sum_{i=1}^{n-1} x_{i}+\frac{\lambda_{n}}{\mu_{n}} x_{n}\right] .
\end{aligned}
$$

This shows (iii).

We are led to illustrate the previous results with some examples where we must have in mind that the condition $x_{k} / \tau_{k} \rightarrow 0(k \rightarrow \infty)$ implies $X \in W_{\tau}^{0}$.

Example 5.3. The condition

$$
\lim _{n \rightarrow \infty} \frac{1}{n} \sum_{k=1}^{n}\left|\frac{x_{k}}{2^{k}}-\frac{7}{4} L\right|=0
$$

for given $L \in \mathbb{C}$ implies $x_{k} \rightarrow L\left(S\left(D_{\left(n / 2^{n}\right)_{n}} C_{1} \Sigma\right)\right)$, that is, for each $\varepsilon>0$

$$
\lim _{n \rightarrow \infty} \frac{1}{n}\left|\left\{k \leq n:\left|\frac{1}{2^{k}} \sum_{i=1}^{k} \sum_{j=1}^{i} x_{j}-L\right| \geq \varepsilon\right\}\right|=0 .
$$

Indeed it is enough to apply Theorem 5.2 (i) with $\lambda_{k}=k, \tau_{k}=2^{k} / k$ and $\mu_{k}=1$ for all $k$. Note that if $x_{k} / 2^{k} \rightarrow 7 L / 4(k \rightarrow \infty)$ then $x_{k} \rightarrow L\left(S\left(D_{\left(n / 2^{n}\right)_{n}} C_{1} \Sigma\right)\right)$.

We can also state the next application.

Example 5.4. If $\lim _{n \rightarrow \infty}(1 / n) \sum_{k=1}^{n}\left|x_{k}\right| / k 2^{k}=0$ then $x_{k} \rightarrow L\left(S\left(D_{\left(2^{-n}\right)_{n}} \Delta C_{1}\right)\right)$, that is for each $\varepsilon>0$

$$
\lim _{n \rightarrow \infty} \frac{1}{n}\left|\left\{k \leq n:\left|\frac{1}{2^{k}}\left(\frac{1}{k}-\frac{1}{k-1}\right) \sum_{i=1}^{k-1} x_{i}+\frac{1}{k} x_{k}\right| \geq \varepsilon\right\}\right|=0 .
$$

This result is a direct consequence of Theorem 5.2 (iii) with $\lambda_{k}=1, \tau_{k}=2^{k}$ and $\mu_{k}=k$ for all $k$. Again note that we have $x_{k} \rightarrow L\left(S\left(D_{\left(2^{-n}\right)_{n}} \Delta C_{1}\right)\right)$ if $x_{k} / k 2^{k} \rightarrow 0(k \rightarrow \infty)$. 
In the following we will use the previous Proposition 4.3 and the expressions of $W_{\tau}^{0}\left(C^{+}(\lambda) \Delta(\mu)\right)=\left[C^{+}, \Delta\right]_{W_{\tau}^{0}}, W_{\tau}^{0}\left(C^{+}(\lambda) C(\mu)\right)=\left[C^{+}, C\right]_{W_{\tau}^{0}}, W_{\tau}^{0}\left(C^{+}(\lambda) C^{+}(\mu)\right)=\left[C^{+}, C^{+}\right]_{W_{\tau}^{0}}$ and $W_{\tau}^{0}\left(\Delta(\lambda) C^{+}(\mu)\right)=\left[\Delta, C^{+}\right]_{W_{\tau}^{0}}$. We now require a lemma which is a direct consequence of Lemma 5.1.

Lemma 5.5. Let $A$ be an infinite matrix. If $X \in w^{0}(A)$ then

$$
x_{k} \rightarrow 0(S(A)) .
$$

we deduce the next results.

Theorem 5.6. (i) Let $\tau \in \Gamma^{+}$and $\lambda \tau \in \Gamma$. If

$$
\lim _{n \rightarrow \infty} \frac{1}{n} \sum_{k=1}^{n} \frac{\left|x_{k}\right|}{\lambda_{k} \tau_{k}} \mu_{k}=0
$$

then $x_{k} \rightarrow 0\left(S\left(D_{1 / \tau} C^{+}(\lambda) \Delta(\mu)\right)\right)$, that is for every $\varepsilon>0$

$$
\lim _{n \rightarrow \infty} \frac{1}{n}\left|\left\{k \leq n:\left|\frac{1}{\tau_{k}} \sum_{i=k}^{\infty} \frac{\mu_{i} x_{i}-\mu_{i-1} x_{i-1}}{\lambda_{i}}\right| \geq \varepsilon\right\}\right|=0
$$

(ii) Let $\tau \in \Gamma^{+}$and $\lambda \mu \tau \in \Gamma$. If

$$
\lim _{n \rightarrow \infty} \frac{1}{n} \sum_{k=1}^{n} \frac{\left|x_{k}\right|}{\lambda_{k} \mu_{k} \tau_{k}}=0
$$

then $x_{k} \rightarrow 0\left(S\left(D_{1 / \tau} C^{+}(\lambda) C(\mu)\right)\right)$, that is for every $\varepsilon>0$

$$
\lim _{n \rightarrow \infty} \frac{1}{n}\left|\left\{k \leq n:\left|\frac{1}{\tau_{k}} \sum_{i=k}^{\infty} \frac{1}{\lambda_{i}}\left(\frac{1}{\mu_{i}} \sum_{j=1}^{i} x_{j}\right)\right| \geq \varepsilon\right\}\right|=0 .
$$

(iii) Let $\tau, \lambda \tau \in \Gamma^{+}$. If

$$
\lim _{n \rightarrow \infty} \frac{1}{n} \sum_{k=1}^{n} \frac{\left|x_{k}\right|}{\lambda_{k} \mu_{k} \tau_{k}}=0
$$

then $x_{k} \rightarrow 0\left(S\left(D_{1 / \tau} C^{+}(\lambda) C^{+}(\mu)\right)\right)$, that is for every $\varepsilon>0$

$$
\lim _{n \rightarrow \infty} \frac{1}{n}\left|\left\{k \leq n:\left|\frac{1}{\tau_{k}} \sum_{i=k}^{\infty} \frac{1}{\lambda_{i}}\left(\sum_{j=i}^{\infty} \frac{x_{j}}{\mu_{j}}\right)\right| \geq \varepsilon\right\}\right|=0 .
$$

(iv) Let $\tau, \tau / \lambda \in \Gamma^{+}$. If

$$
\lim _{n \rightarrow \infty} \frac{1}{n} \sum_{k=1}^{n} \frac{\lambda_{k}\left|x_{k}\right|}{\mu_{k} \tau_{k}}=0
$$

then $x_{k} \rightarrow 0\left(S\left(D_{1 / \tau} \Delta(\lambda) C^{+}(\mu)\right)\right)$, that is for every $\varepsilon>0$

$$
\lim _{n \rightarrow \infty} \frac{1}{n}\left|\left\{k \leq n: \frac{1}{\tau_{k}}\left|\left(\lambda_{k}-\lambda_{k-1}\right) \sum_{i=k-1}^{\infty} \frac{x_{i}}{\mu_{i}}+\frac{\lambda_{k}}{\mu_{k}} x_{k}\right| \geq \varepsilon\right\}\right|=0 .
$$


Proof. (i) Condition (15) implies $X \in W_{\lambda \tau / \mu}^{0}$ and by Proposition 4.3 and Remark 4.4 since $\tau \in \Gamma^{+}$ and $\lambda \tau \in \Gamma$ we have $W_{\lambda \tau / \mu}^{0}=W_{\tau}^{0}\left(C^{+}(\lambda) \Delta(\mu)\right)$ and $X \in W_{\tau}^{0}\left(C^{+}(\lambda) \Delta(\mu)\right)$. Now it can be easily seen that

$$
\left[D_{1 / \tau} C^{+}(\lambda) \Delta(\mu)\right]_{n}=\frac{1}{\tau_{n}} \sum_{i=n}^{\infty} \frac{\mu_{i} x_{i}-\mu_{i-1} x_{i-1}}{\lambda_{i}},
$$

so by Lemma 5.5 with $A=D_{1 / \tau} C^{+}(\lambda) \Delta(\mu)$ we conclude $x_{k} \rightarrow 0\left(S\left(D_{1 / \tau} C^{+}(\lambda) \Delta(\mu)\right)\right)$. This shows (i).

(ii) Here condition (17) means $X \in W_{\lambda \mu \tau}^{0}$ and by Proposition 4.3 and Remark 4.4 since $\tau \in \Gamma^{+}$and $\lambda \mu \tau \in \Gamma$ we have $W_{\lambda \mu \tau}^{0}=W_{\tau}^{0}\left(C^{+}(\lambda) C(\mu)\right)$ and $X \in W_{\tau}^{0}\left(C^{+}(\lambda) C(\mu)\right)$. Now since

$$
\left[D_{1 / \tau} C^{+}(\lambda) C(\mu)\right]_{n}=\frac{1}{\tau_{n}} \sum_{i=n}^{\infty} \frac{1}{\lambda_{i}}\left(\frac{1}{\mu_{i}} \sum_{j=1}^{i} x_{j}\right),
$$

by Lemma 5.5 where $A^{\prime}=D_{1 / \tau} C^{+}(\lambda) C(\mu)$, we conclude $x_{k} \rightarrow 0\left(S\left(D_{1 / \tau} C^{+}(\lambda) C(\mu)\right)\right)$. So we have shown (ii).

(iii) can be obtained reasoning as above with $A^{\prime \prime}=D_{1 / \tau} C^{+}(\lambda) C^{+}(\mu)$ and so $x_{k} \rightarrow 0$ $\left(S\left(D_{1 / \tau} C^{+}(\lambda) C^{+}(\mu)\right)\right)$.

(iv) can also be obtained similarly. It is enough to put $A^{\prime \prime \prime}=D_{1 / \tau} \Delta(\lambda) C^{+}(\mu)$. An elementary calculation gives

$$
\left[A^{\prime \prime \prime} X\right]_{k}=\frac{1}{\tau_{k}}\left[\left(\lambda_{k}-\lambda_{k-1}\right) \sum_{i=k-1}^{\infty} \frac{x_{i}}{\mu_{i}}+\frac{\lambda_{k}}{\mu_{k}} x_{k}\right]
$$

and we conclude that $x_{k} \rightarrow 0\left(S\left(D_{1 / \tau} \Delta(\lambda) C^{+}(\mu)\right)\right)$, that is (21).

We can state the next example

Example 5.7. for each $\varepsilon>0$ and for every $X \in W_{3 / 2}^{0}$ we have $x_{k} \rightarrow 0\left(S\left(D_{\left(2^{n}\right)_{n}} \Sigma^{+} C\left(\left(3^{n}\right)_{n}\right)\right)\right)$, that is

$$
\lim _{n \rightarrow \infty} \frac{1}{n}\left|\left\{k \leq n:\left|2^{k} \sum_{i=1}^{\infty} \frac{1}{3^{i}}\left(\sum_{j=1}^{i} x_{j}\right)\right| \geq \varepsilon\right\}\right|=0 .
$$

It is enough to apply Theorem 5.6 (ii) with $\tau_{k}=2^{-k}, \mu_{k}=3^{k}$ and $\lambda_{k}=1$ for all $k$. So if $(2 / 3)^{k} x_{k} \rightarrow 0(k \rightarrow \infty)$ then (22) holds.

We also have the next example.

Example 5.8. From Theorem 5.6 (iii) with $\lambda_{k}=\mu_{k}=k$ and $\tau_{k}=2^{-k}$ the condition

$$
\lim _{n \rightarrow \infty} \frac{1}{n} \sum_{k=1}^{n} 2^{k} \frac{\left|x_{k}\right|}{k^{2}}=0
$$


implies $x_{k} \rightarrow 0\left(S\left(D_{\left(2^{n}\right)_{n}} C_{1} C_{1}^{+}\right)\right)$that is, for each $\varepsilon>0$

$$
\lim _{n \rightarrow \infty} \frac{1}{n}\left|\left\{k \leq n:\left|2^{k} \sum_{i=k}^{\infty} \frac{1}{i}\left(\sum_{j=i}^{\infty} \frac{x_{j}}{j}\right)\right| \geq \varepsilon\right\}\right|=0 .
$$

As in the previous cases (23) holds if $2^{k} x_{k} / k^{2} \rightarrow 0(k \rightarrow \infty)$.

\section{References}

[1] ÇOLAK, R., Lacunary strong convergence of difference sequences with respect to a modulus, Filomat, 17 (2003), 9-14.

[2] DE Malafosse, B., On some BK space, Int. J. of Math. and Math. Sc., 28 (2003), 17831801.

[3] DE Malafosse, B., On the set of sequences that are strongly $\alpha$-bounded and $\alpha$ convergent to naught with index $p$, Seminario Matematico dell'Università e del Politecnico di Torino, 61 (2003), 13-32.

[4] DE Malafosse, B., Calculations on some sequence spaces, Int. J. of Math. and Math. Sc., 31 (2004), 1653-1670.

[5] De Malafosse, B. And Malkowsky, E., The Banach algebra $\left(w_{\infty}(\lambda), w_{\infty}(\lambda)\right)$, in press Far East Journal Math.

[6] De Malafosse, B. ANd RakočEvić, V., Matrix Transformations and Statistical convergence, Linear Algebra and its Applications, 420 (2007), 377-387.

[7] FAST, H., Sur la convergence statistique, Colloq. Math., 2 (1951), 241-244.

[8] FRIDY, J.A., On statistical convergence, Analysis, 5 (1985), 301-313.

[9] FrIDY, J.A., Statistical limit points, Proc. Amer. Math. Soc., 118 (1993), 1187-1192.

[10] Fridy, J.A. AND ORhan, C., Lacunary statistical convergence, Pacific J. Math., 160 (1993), 43-51.

[11] Fridy, J.A. AND ORHAN, C., Statistical core theorems, J. Math. Anal. Appl., 208 (1997), $520-527$.

[12] Maddox, I.J., On Kuttner's theorem, J. London Math. Soc., 43 (1968), 285-290.

[13] Maddox, I.J., Elements of Functionnal Analysis, Cambridge University Press, London and New York, 1970. 
[14] Malkowsky, E., The continuous duals of the spaces $c_{0}(\Lambda)$ and $c(\Lambda)$ for exponentially bounded sequences $\Lambda$, Acta Sci. Math (Szeged), 61, (1995), 241-250.

[15] Malkowsky, E. AND RakočEvić, V., An introduction into the theory of sequence spaces and measure of noncompactness, Zbornik radova, Matematčki institut SANU, 9 (17) (2000), 143-243.

[16] Steinhaus, H., Sur la convergence ordinaire et la convergence asymptotique, Colloq. Math., 2 (1951), 73-74.

[17] Wilansky, A., Summability through Functional Analysis, North-Holland Mathematics Studies, 85, 1984. 